\title{
Power Losses Calculations in Windings of Gapped Magnetic Components:
}

\author{
The i2D Method Applied to Flyback Transformers
}

\author{
Fermin A. Holguin, Roberto Prieto, Rafael Asensi, Jose A. Cobos
}

\begin{abstract}
The improved 2-D equivalent analytical calculation method to estimate conductive losses in gapped magnetic components in a wide range of frequencies is extended to the calculations of conduction losses in flyback transformers. The i2D method, that is applicable to power inductor, is extended is extended to the winding loss calculation in gapped transformers, such as flyback transformers, by means of harmonic decomposition of the current though the windings that allows the proximity field calculation and, afterward, the estimation of the losses in the windings.
\end{abstract}

The method is used to evaluate conduction losses in flyback transformers for switched mode power supplies (SMPS) and the results are compared with finite element analysis (FEA) and measurements.

Keywords-flyback transformers; winding loss; magnetic components; gap

\section{INTRODUCTION}

As the effect of the air gap in the winding loss is not considered in commonly used methods [1-6], the calculation of conduction losses in windings of gapped magnetic components cannot be accurately estimated using conventional approaches. As a consequence, numerical analysis tools, such as FEA tools, are often required for the component characterization [7-9] with the disadvantage of larger design time and the difficulty to implement iterative optimization processes.

Many methods used for the calculation of conduction loss in magnetic components are based on the winding's loss separation concept which allows the independent calculation of the effects of the local and external induced eddy-currents, and then the total winding loss is found to be the superposition of all the considered loss mechanisms (dc, skin, proximity). This concept conveniently allows the calculation of conduction loss by "modules" offering the possibility to evaluate each component of the loss in a different way, giving us flexibility in choosing a desired approach to estimate the different contributions of loss mechanism.

This concept is used independently of the assumed geometry of the fields in both 1-D and 2-D structures. In 1-D structures (field considered 1-dimensional), this is done calculating skin and proximity effects separately thanks to their orthogonality [2]-[3], [5], [11]-[15] and [24]-[25] but cannot be used in gapped magnetics where a 2-D component of the magnetic field, which is the fringing field of the air gap, shall be considered and introduced in the calculations. In this kind of structures, the field calculation is an important "intermediate" step to estimate the winding loss.

Several solutions in the state of the art can be used for the estimation of wingding loss in gapped magnetic components [10]-[12], [17]-[28]. The solution presented in [10] accounts for gapped magnetic components with planar conductors and provides analytical expressions to determine the magnetic field at a given position from the gap and. The calculation of winding loss focused in the optimization of gapped inductors is handled in [11], while [12] define an improved method for the field calculation used in [11] and, therefore, an improvement in the conductive loss calculations. This approach uses the mirror image technique and allows a tradeoff between accuracy and computational effort by choosing an appropriate number of images. What is really interesting about this method is that the core influence is "eliminated" replacing the air gap with an equivalent current sheet (that "produces" an equivalent magnetic field as the air gap). [17]-[19], [23] and [28] deal with the calculation of winding loss in gapped inductors using a 2-D representation of the magnetic field $H$ at an arbitrary position inside the window area of the core. As the magnetic field $H$ is evaluated by means of the magnetic potential (in the form of, either, the vector or scalar potential) that fulfils the Laplace equation, this approach might result in complex equations that can be very difficult to implement (if its integration in a design and optimization tool is desired). A general procedure to extend, or apply, the loss separation concept to multi-winding components (that might have different currents in each) is explained in [21] and [20] and it is shown how to calculate the desired parameters using a winding loss/resistance matrix. In [13] a simple solution based on the well-known Dowell's method [1] is proposed, but it can be properly applied only in components whose windings are arranged in a "layered" structure which is not the case of winding structures in typical flyback transformers. In [15] an analytical 2D equivalent method based on the superposition of losses mechanisms (skin, 
gap and proximity) in the winding of the magnetic component based on a simple calculation of the fields is proposed.

In this paper, a procedure to extend the i2D analytical method [15] to the calculation of winding loss in gapped transformers, such as flyback transformers, is shown and compares the results with FEA calculations. Afterward, the method is used to estimate losses in the transformer of a flyback converter and the results are compared with measurements.

\section{THE I2D METHOD}

In [15] the power loss per unit length of a cylindrical conductor under a transverse magnetic field is calculated by means of expression (1) from [16], where ber, bei, ber' and $b e i$ ' are, respectively, the real and imaginary part of the Bessel functions of first kind and their derivatives, $\sigma$ and $\mu$ the conductivity and permeability of the conductive material, $r_{0}$ is the radius of the conductor and $H_{0}$ is the magnitude of the transverse field to the conductor.

$$
P(W / m)=\frac{\pi \mu^{2}}{\sigma} k r_{0} H_{0}^{2} \frac{\operatorname{ber}\left(k r_{0}\right) b e i^{\prime}\left(k r_{0}\right)-\operatorname{ber}^{\prime}\left(k r_{0}\right) b e i\left(k r_{0}\right)}{\mu^{2}\left[\operatorname{ber}\left(k r_{0}\right)\right]^{2}+\mu^{2}\left[\operatorname{bei}\left(k r_{0}\right)\right]^{2}}
$$

Where $k=\sqrt{w \sigma \mu}$ and $w$ is the angular frequency. Since the fringing field due to the air gap in a magnetic component (fig. 1), Hg, is considered to be two-directional in the plain $r z$ in cylindrical coordinates, any conductor affected by this fringing field will be exposed to its two components, $H r$ and $\mathrm{Hz}$, both transverse to the conductor. The field components of $\mathrm{Hg}$ can be calculated, according to [10], with (2) and (3) for $r$ and $z$ components respectively, where $l g$ is half of the air gap length, $r$ and $z$ are the cylindrical coordinates respect of the origin (see figure 1), $m$ is equal to zero if $r^{2}+z^{2}>l_{g}^{2}$ and equal to 1 if $\mathrm{r}^{2}+\mathrm{z}^{2}<\mathrm{l}_{\mathrm{g}}^{2}$ and $H_{g} \cong 0.9 N I / 2 l_{g}$ with $N$ and $I$ equal to the number of turns and peak current through the winding, respectively.

$$
\begin{gathered}
H_{r}(r, z)=\frac{H_{g}}{2 \pi} \ln \left[\frac{r^{2}+\left(z-l_{g}\right)^{2}}{r^{2}+\left(z+l_{g}\right)^{2}}\right] \\
H_{z}(r, z)=-\frac{H_{g}}{\pi}\left[\tan ^{-1}\left(\frac{2 r l_{g}}{r^{2}+z^{2}-l_{g}^{2}}\right)+m \pi\right]
\end{gathered}
$$

Since proximity and gap effects, are actually the same effect (external fields), they can be calculated using the same expression. If we assume that fringing and proximity fields are static and independent (and superposition theorem can be used), the Biot-Savart law (expression 4) can be used to calculate the magnetic field that is affecting a single conductor of length $L$ due to the electric current $I$ flowing through a near conductor at a position $x$ by means of expression (5) (fig. 1), where $\hat{x}$ is an unitary vector in the direction of $x$.

$$
\begin{gathered}
d \vec{H}=\frac{1}{4 \pi} \frac{I d \vec{l} \times \hat{x}}{x^{2}} \\
\vec{H}=-\frac{I}{2 \pi x} \frac{L}{\sqrt{L^{2}+x^{2}}}(\hat{x})
\end{gathered}
$$

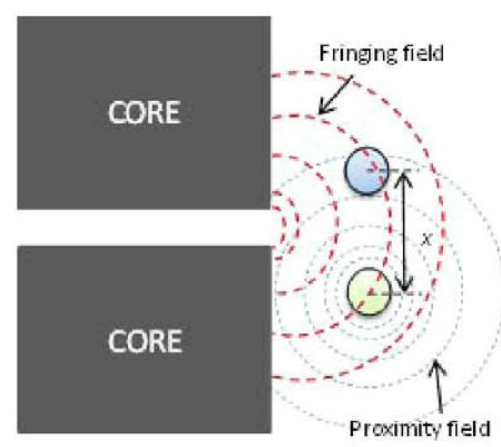

Figure 1. Representation of the influence of both fringing and proximity fields over a round conductor in a gapped magnetic component.

At this point, $P r$ and $P z$, that are the proximity loss components, can be calculated assuming $H_{0}$ as the sum of the corresponding components of the fringing and proximity fields in (1). Then, the total loss per unit length of the considered conductor can be obtained as the sum of (6), and (7) multiplied by the square of the RMS current which are the proximity and skin effects contributions respectively.

$$
\begin{gathered}
P_{g}(W / m)=P_{r}+P_{Z} \\
R_{S}(\Omega / m)=\operatorname{Re}\left[\frac{j^{\frac{3}{2}} k}{2 \pi r_{0} \sigma} \frac{J_{0}\left(j^{\frac{3}{2}} k r_{0}\right)}{J_{1}\left(j^{\frac{3}{2}} k r_{0}\right)}\right]
\end{gathered}
$$

\section{A. I2D Limitations}

The $12 \mathrm{D}$ method has two important limitations that need to be considered:

- The expressions (2) and (3), which are used to calculate the fringing field due to the air gap at a given position of the window breath, are limited to components with a symmetric gap with respect to its core. This means that they can only be used in concentric geometries with a central air gap placed in its central leg (such as RM, EE and ETD like structures). This approach, however, could be extended to components with distributed air gap as long as it position remains symmetric with respect to the core. In this case $H_{g}$ and $H_{g}$, which are the magnitude of the reference sources (in central and external legs) of the fringing field, must be calculated in a different way (for instance, using a reluctance model).

- Since it is a simplified method, where the fringing and proximity fields are considered to be "static and independent" and are calculated separately, an error, especially at high frequencies, is introduced in the calculation of the fields and, thus, in the calculation of losses. Moreover, the main equation which uses Bessel functions that are not very accurate at high frequencies when it is applied to close packed windings, expression (1), which is valid for a homogeneous magnetic field, is used in an application where the external field is not uniform and this is, also, a source of errors in the estimations. 


\section{I2D EXTENSION TO GAPPED TRANSFORMERS}

A natural way to use the $12 \mathrm{D}$ method in flayback transformers is the calculation of the loss in each winding by separate at each frequency point. For instance, consider the case of figure 2. During T1, when primary winding is conducting $I_{1}$, the power loss can be found evaluating the proximity loss (external fields) in windings $W_{1}$ and $W_{2}$, calculated by means of expression (1), plus the skin effect contribution (internal fields) in $W_{1}$. Since $W_{2}$ is not conducting it has no skin effect contribution in the loss during this state. The same occurs during $\mathrm{T} 2$, where $W_{1}$ is not conducting and $W_{2}$ is carrying $I_{2}$. In this state the loss is the contribution of the proximity field in $W_{1}$ and $W_{2}$ plus the skin contribution only in $W_{2}$. Then the total loss is the sum of $P_{1}$ and $P_{2}(10)$.

$$
\begin{gathered}
P_{1}=P_{P, W 1}+P_{P, W 2}+P_{S, W 1} \\
P_{2}=P_{P, W 1}+P_{P, W 2}+P_{S, W 2} \\
P_{\text {Total }}=P_{1}+P_{2}
\end{gathered}
$$

This is not different than the application in power inductors. In fact it's just like applying the i2D method in two inductors (but considering the eddy losses in additional non-excited conductors). This leads to the necessity of applying i2D two times in the same component (at each state).

In order to avoid this "double application", the currents through the windings can be considered as flowing simultaneously by means of the analysis in frequency domain. This is carried out through the harmonic decomposition of the currents in the windings and calculating the proximity loss, as well as the skin loss, at any frequency point by means of the calculation of the fields according to the corresponding values of the primary and secondary currents at the same time. This way the fringing field source, $H_{g}$, can be assumed as the same for both windings, since $N_{1} I_{1}=N_{2} I_{2}$, and is calculated according to the peak value of the current at the considered harmonic (using either $I_{1}$ or $I_{2}$ for the determination of $H_{g}$ ). The field components, $\mathrm{Hr}$ and $\mathrm{Hz}$, are evaluated with (2) and (3) respectively. The proximity fields are calculated with (5) using the corresponding current for the considered turn (where the direction of the currents is considered as a phase information in the frequency domain).

The analytical results of components in the fig. 3 are compared with FEA in table 1. It can be seen that the $12 \mathrm{D}$ analytical model that was used for computing the winding loss shows very good results with respect to FEA calculations, which is considered a very accurate solution. Also, the results using the skin depth approach (which does not include the effect of the fringing field) are included in the table in order to compare with a commonly used calculation method (in these results, the proximity effect is not considered because the commonly used 1D approach cannot be applied to the given 2D winding structures).
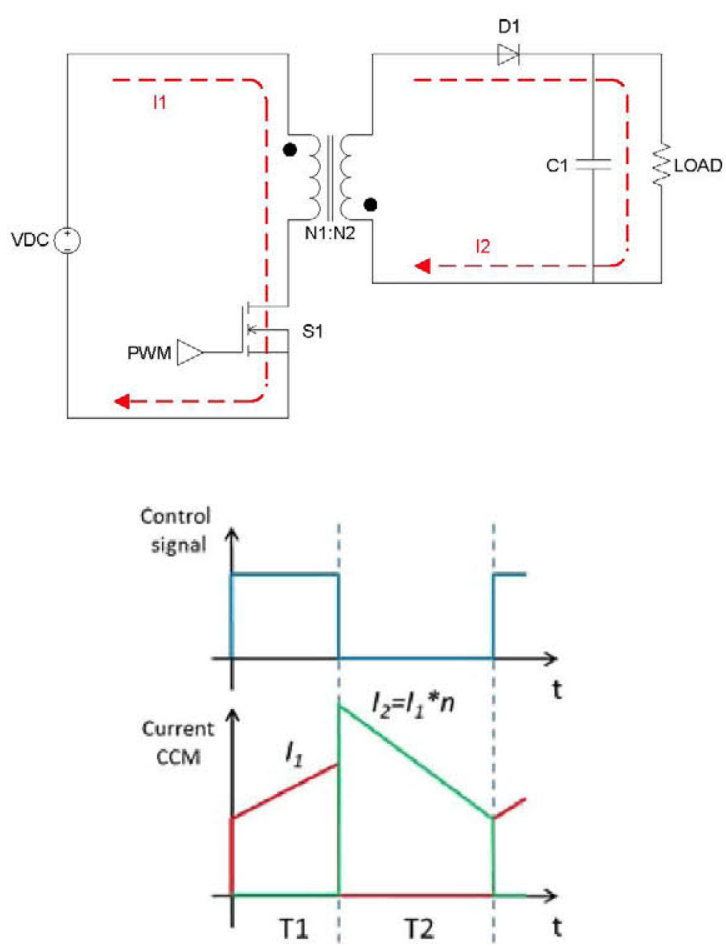

Figure 2. Currents through the windings of a flyback transformer. Time domain.

\section{RESUltS}

The results of the application of the i2D model in flyback transformers are compared with FEA and measurements on a SMPS prototype.

In order to compare with FEA, consider the transformers shown in figure 3 as three possible designs of a transformer for a hypothetical SMPS working in continuous conduction mode at $400 \mathrm{kHz}$ (see the waveform of the currents in figures $2 \mathrm{~d}$ an 2e) with input and output voltages of $24 \mathrm{~V}$ and $80 \mathrm{~V}$ respectively and $160 \mathrm{~W}$ of nominal power. The transformers were modeled in the FEA tool ANSYS MAXWELL using the structure transformation described in [17] and simulated at different frequencies that correspond to the first 15 harmonics of the currents. A copper conductivity of $45.249 \times 10^{6} \mathrm{~S} / \mathrm{m}$ was used for the conductors.

The analytical results for components $3 \mathrm{a}, 3 \mathrm{~b}$ and $3 \mathrm{c}$ are compared with FEA in table 1. It can be seen that the $\mathrm{i} 2 \mathrm{D}$ analytical model that was used for computing the winding loss shows very good results with respect to FEA calculations, which is considered a very accurate solution. Also, the results using the skin depth approach (which does not include the effect of the fringing field) are included in the table in order to compare with a commonly used calculation method (in these results, the proximity effect is not considered because the commonly used 1D approach cannot be applied to the given 2D winding structures). 


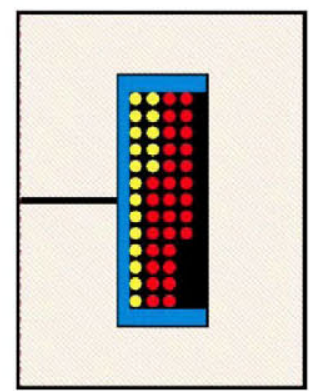

(a)

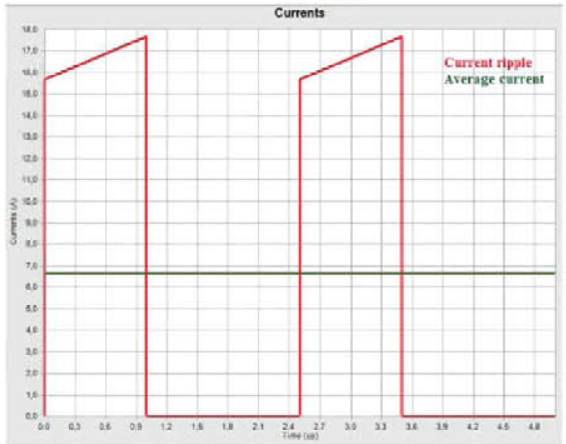

(d)

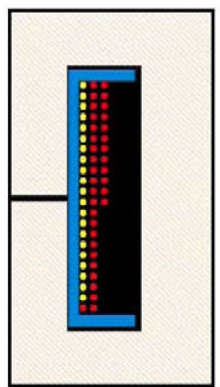

(b)

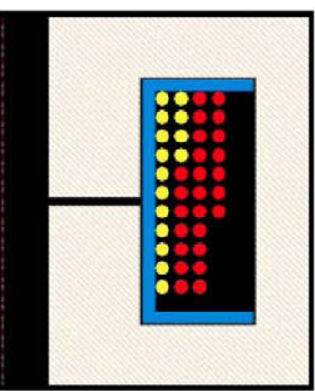

(c)

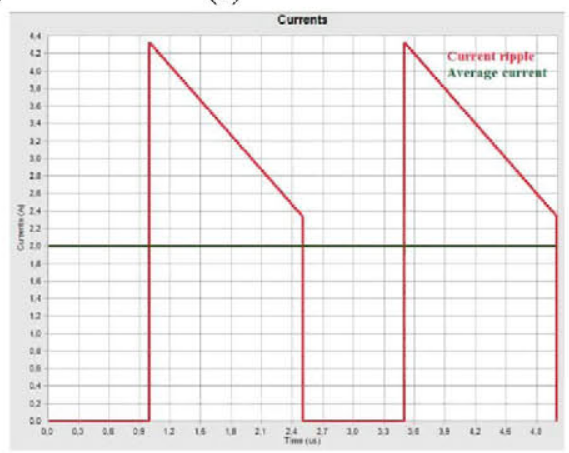

(e)

Figure 3. Axisymmetric representation of flyback transformers with (a) $514.48 \mu \mathrm{m}$ air gap, 6 AWG20 turns (3 parallel) in primary and 30 AWG20 turns in secondary in a 3C95 RM12/I core, (b) $588.17 \mu \mathrm{m}$ air gap, 7 AWG20 turns (3 parallel) in primary and 35 AWG20 turns in secondary in a 3C95 EC41 core, (c) $501.04 \mu \mathrm{m}$ air gap, 5 AWG20 turns (3 parallel) in primary and 25 AWG20 turns in secondary in a 3C95 P36/22 core, and (d) and (e) are the hypothetic current waveforms of the primary and secondary windings of the transformer respectively.

Table 1. Comparison between analytical and FEA calculated winding loss of transformers in fig. 2.

\begin{tabular}{cccc}
\hline \multirow{2}{*}{ Component } & \multicolumn{3}{c}{ Calculated Power loss (W) } \\
\cline { 2 - 4 } & FEA & $\begin{array}{c}\text { Analytical } \\
\text { (proposed) }\end{array}$ & $\begin{array}{c}\text { Analytical } \\
\text { (skin depth approach) }\end{array}$ \\
$\mathbf{2 a}$ & 10.44 & 10.25 & 1.77 \\
$\mathbf{2 b}$ & 7.89 & 7.53 & 1.89 \\
\hline $\mathbf{2 c}$ & 9.41 & 9.16 & 1.76 \\
\hline
\end{tabular}

A further comparison against measurements on a flyback converter prototype has been done. Three different transformers were built (fig. 4) and used in the flyback prototype of fig. 5a. The characteristics of the transformers as well as the operating conditions and measurements in the converter are stated in table $2,3 \mathrm{a}$ and $3 \mathrm{~b}$ respectively. The duty cycle has been fixed (around 15\% and 50\%) in all tests and is also specified in the operating condition in table $3 \mathrm{a}$. The calculated results (by means of the skin depth approach and the proposed method) are compared with measured values in table $3 \mathrm{~b}$.

The operating frequency is $100 \mathrm{kHz}$. The driving signal for the main switch is generated in a digital control board which is supplied with a different power source. Since the focus is to measure the power loss in the transformers, no special care was taken in the design of the converter. A dissipative clamping circuit is used for limiting the voltage spikes in the transformers $(\mathrm{Cs}=880 \mathrm{pF}$ and $\mathrm{Rs}=66 \Omega)$.

\section{A. Measurement Setup and Procedure}

In order to measure the power loss in the transformers we followed the procedure detailed bellow:

- For a given load in the converter, the input and output power are measured using precision meters while an infrared camera is used to register the temperature of the semiconductors (main switch, the clamping diode and output diode) and the clamping resistor. The total loss in the converter is difference between the measured input and output power.

Since a regulated source is used for the input voltage, no input capacitor is used in order to avoid additional loss due to the corresponding ESR. Also the required output capacitance, as well as the clamping capacitance, is 
obtained stacking a big number of parallel capacitors (also in order to reduce ESR).

- The semiconductors and clamping resistor are later characterized in order to subtract the corresponding loss from the measured values. To do this, the information taken from the thermal monitoring is used (every component is characterized in order to define their associated losses at a given temperature corresponding to the considered operating points). This is done pushing a controlled de current through the device to make it reach the same temperature that was observed during operation, associating a given temperature with a specific power loss.

- Then the power loss in the transformer (that includes the core loss) is assumed to be the difference between the total power loss and losses in the semiconductors and clamping circuit.

- The core loss is later taken out from the obtained value by means of the subtraction of an analytically calculated core loss [29] using (12), where $k, \alpha$ and $\beta$ are the Steinmetz parameters of the used magnetic materials (taken from datasheet). The waveform of the flux density is determined according to the measured values of the currents, or voltages, in the transformer (magnetizing current).

$$
\begin{gathered}
\bar{P}(W)=\frac{1}{T} \int_{0}^{T} V * I d t \\
\bar{P}_{v}=\frac{1}{T} \int_{0}^{T} k_{i}\left|\frac{d B}{d t}\right|^{\alpha}(\Delta B)^{\beta-\alpha} d t
\end{gathered}
$$

With

$$
k_{i}=\frac{k}{(2 \pi)^{\alpha-1} \int_{0}^{2 \pi}|\cos \theta|^{\alpha} 2^{\beta-\alpha} d \theta}
$$

\begin{tabular}{|c|c|c|c|c|}
\hline \multirow{2}{*}{ Component } & \multirow{2}{*}{$\begin{array}{c}\text { Core } \\
\text { Material }\end{array}$} & \multirow{2}{*}{$\begin{array}{c}\text { Gap } \\
\text { length } \\
(\mathrm{mm})\end{array}$} & \multicolumn{2}{|c|}{ Turns/Parallel } \\
\hline & & & Primary & Secondary \\
\hline T1 & $\begin{array}{c}\mathrm{RM} 8 / \mathrm{I} \\
3 \mathrm{~F} 3\end{array}$ & 0.40 & $\begin{array}{c}20 / 1 \\
\text { AWG23 }\end{array}$ & $\begin{array}{c}3 / 3 \\
\text { AWG23 }\end{array}$ \\
\hline T2 & $\begin{array}{c}\mathrm{RM} 8 / \mathrm{I} \\
3 \mathrm{~F} 3\end{array}$ & 0.72 & $\begin{array}{c}26 / 1 \\
\text { AWG25 }\end{array}$ & $\begin{array}{c}4 / 3 \\
\text { AWG25 }\end{array}$ \\
\hline T3 & $\begin{array}{c}\text { RM10 } \\
\text { N41 }\end{array}$ & 0.44 & $\begin{array}{c}12 / 1 \\
\text { AWG23 }\end{array}$ & $\begin{array}{c}2 / 3 \\
\text { AWG19 }\end{array}$ \\
\hline
\end{tabular}

Table 2. Characteristics of the measured transformers.

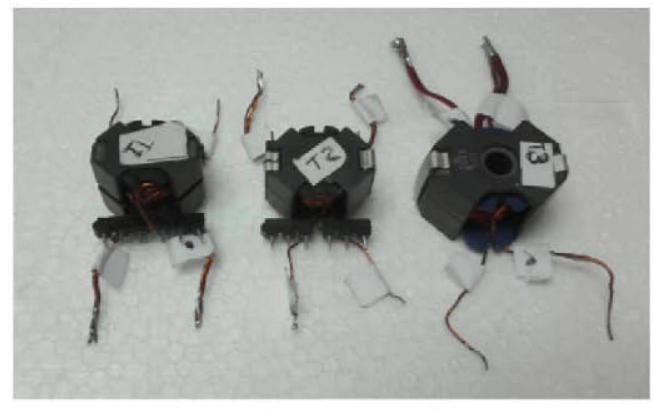

Figure 4. Measured transformers.

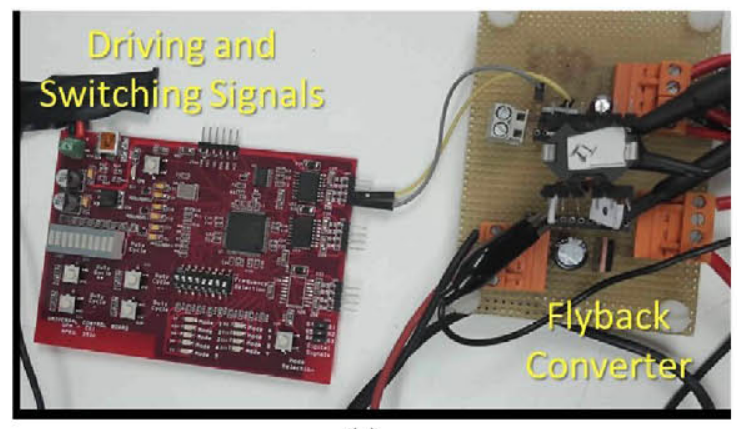

(a)

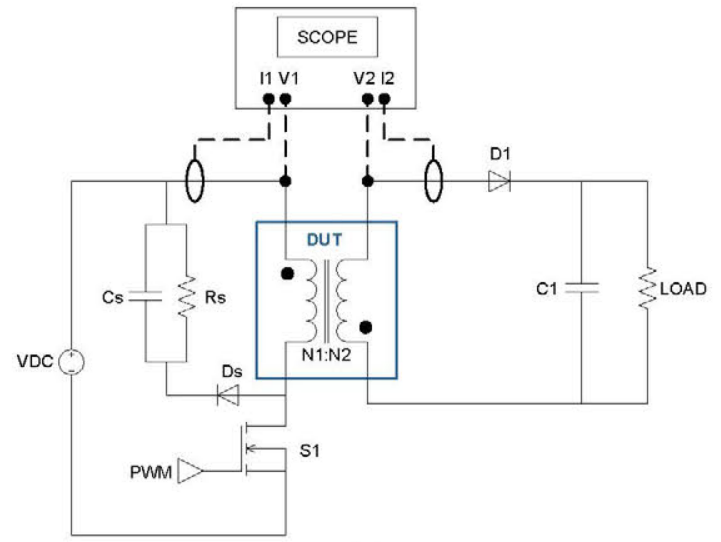

(b)

Figure 5. Flyback converter used for measuring winding loss in gapped transformers. (a) Flyback converter prototype and the digital control card for the driving signals and (b) setup for measuring the transformers currents in the flyback converter.
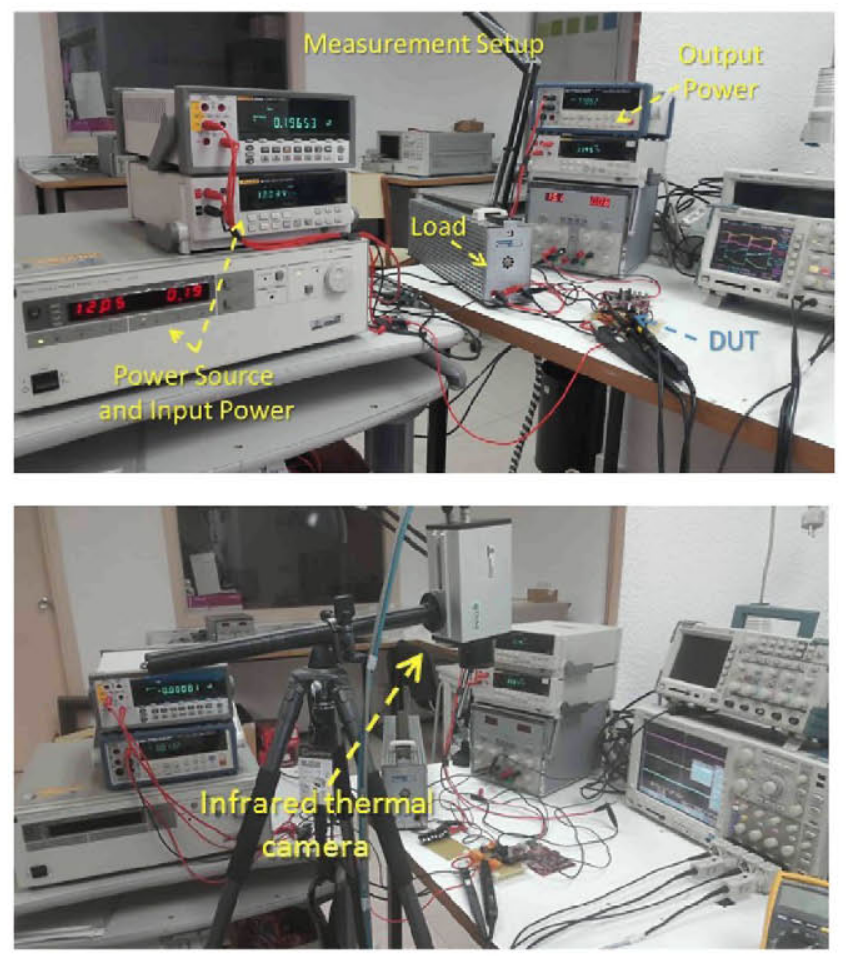

Figure 6. Setup for the loss measurement in the flyback transformer. 
Table 3a. Operating conditions of measured transformers in the flyback converter prototype.

\begin{tabular}{|c|c|c|c|c|c|c|}
\hline \multirow{2}{*}{ DUT } & \multirow{2}{*}{$\begin{array}{c}\text { Duty } \\
\text { Cycle }(\%)\end{array}$} & \multicolumn{2}{|c|}{ INPUT } & \multicolumn{2}{|c|}{ OUTPUT } & \multirow{2}{*}{ Temperature $\left({ }^{\circ} \mathrm{C}\right)$} \\
\hline & & Voltage (V) & Current (A) & Voltage (V) & Current (A) & \\
\hline \multirow{3}{*}{$\mathrm{T} 1$} & 50 & 40.450 & 0.800 & 1.750 & 3.090 & $\begin{array}{l}\text { MOSFET: } 113.2 \text { DIODE: } 61.0 \\
\text { Rs: } 104.4 \text { Ds: } 46.8\end{array}$ \\
\hline & 50 & 28.300 & 0.600 & 1.260 & 2.230 & $\begin{array}{c}\text { MOSFET: } 56.5 \text { DIODE: } 52.8 \\
\text { Rs: } 70.7 \text { Ds: } 39.4\end{array}$ \\
\hline & 15 & 48.150 & 0.056 & 1.160 & 0.520 & $\begin{array}{c}\text { MOSFET: } 32.0 \text { DIODE: } 34.1 \\
\text { Rs: } 37.3 \text { Ds: } 31.8\end{array}$ \\
\hline \multirow{3}{*}{$\mathrm{T} 2$} & 50 & 39.200 & 0.790 & 2.200 & 2.860 & $\begin{array}{l}\text { MOSFET: } 99.0 \text { DIODE: } 59.0 \\
\text { Rs: } 98.8 \text { Ds: } 43.9\end{array}$ \\
\hline & 50 & 27.110 & 0.580 & 1.560 & 2.030 & $\begin{array}{c}\text { MOSFET: } 53.7 \text { DIODE: } 51.0 \\
\text { Rs: } 65.4 \text { Ds: } 38.6\end{array}$ \\
\hline & 15 & 48.200 & 0.059 & 1.220 & 0.562 & $\begin{array}{c}\text { MOSFET: } 31.0 \text { DIODE: } 34.0 \\
\text { Rs: } 36.5 \text { Ds: } 30.6\end{array}$ \\
\hline \multirow{2}{*}{ T3 } & 50 & 28.000 & 0.780 & 1.100 & 1.950 & $\begin{array}{l}\text { MOSFET: } 141.8 \text { DIODE: } 50.2 \\
\text { Rs: } 77.7 \text { Ds: } 38.2\end{array}$ \\
\hline & 15 & 48.130 & 0.113 & 0.860 & 1.020 & $\begin{array}{c}\text { MOSFET: } 33.8 \text { DIODE: } 39.2 \\
\text { Rs: } 45.2 \text { Ds: } 31.5\end{array}$ \\
\hline
\end{tabular}

Table 3b. Measured values in the flyback converter prototype.

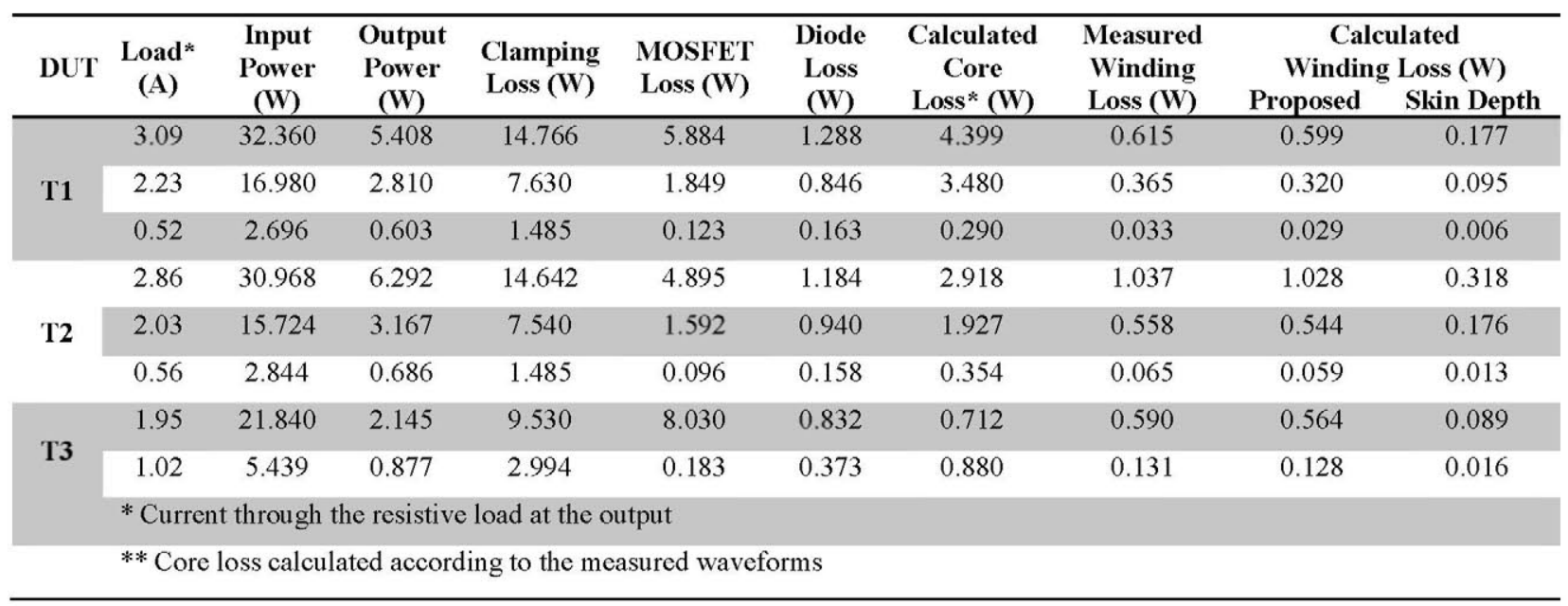

\section{CONCLUSION}

The improved analytical method for calculating winding loss in gapped magnetic components is extended to the calculation of conductive losses in gapped transformers through the harmonic decomposition of the currents and assuming them, at any frequency point, flowing through both windings at the same time. As the fields are considered "independent and static" in order to use superposition and BiotSavart law, the same limitations regarding the accuracy that are present in the $\mathrm{i} 2 \mathrm{D}$ method remain in this extension.

The analytical results are compared with numerical calculations (FEA) and measurements from which the validity of the calculation method can be derived.

\section{REFERENCES}

[1] P.L. Dowell, "Effects of eddy currents in transformer windings", Proceedings of the IEE, vol. 113, no. 8, pp. 1387-1394, Aug. 1966.

[2] Xi Nan and Charles R. Sullivan, "An improved calculation of proximity effect loss in high frequency windings of round conductors", 34th Annual IEEE Power Electronics Specialists Conference, 2003, vol. 2, pp. 853-860.

[3] Xi Nan and Charles R. Sullivan, "Simplified high-accuracy calculation of eddy-current loss in round-wire windings", 35th Annual IEEE Power Electronics Specialists Conference, 2004, vol. 2, pp. 873 - 879.

[4] F. Robert, P. Mathys and J. P. Schauwers "A closed-form formula for 2D ohmic losses calculations in SMPS transformer foils", IEEE Transactions on Power Electronics, vol. 16, pp. 437-444, May 2001.

[5] J. A. Ferreira, "Improved analytical modeling of conductive losses in magnetic components", IEEE Transactions on Power Electronics, vol. 9, no. 1, pp. 127-31, Jan. 1994. 
[6] Mohammad Etemadrezaei and Srdjan M. Lukic "Equivalent complex permeability and conductivity of litz wire in wireless power transfer systems", IEEE Energy Conversion Congress and Expositions, 2012, pp. 3833-3840

[7] R. Asensi, J.A. Cobos, O. García, R. Prieto and J. Uceda "A full procedure to model high frequency transformer windings", 25th Anmual IEEE Power Electronics Specialists Conference, 1994, vol. 2, pp. 856863.

[8] R. Asensi, R. Prieto, J.A. Cobos and J. Uceda "Modeling high-frequency multiwinding magnetic components using finite-element analysis", IEEE Transactions on Magnetics, vol. 43, no. 10, pp. 3840-3850, 2007.

[9] R. Prieto, R. Asensi, C. Fernandez, J.A. Oliver and J.A. Cobos, "Bridging the gap between FEA field solution and the magnetic component model", IEEE Transactions on Power Electronics, vol. 22, no. 3, pp. 943-951, 2007.

[10] Waseem A. Roshen "Fringing Field Formulas and Winding Loss Due to an Air Gap", IEEE Transactions on Magnetics, vol. 43, no. 8, pp. 3387-3394, August 2007.

[11] Jiankun $\mathrm{Hu}$ and Charles R. Sullivan "Optimization of shapes for roundwire high-frequency gapped-inductor windings", 33th Annual IEEE Industry Applications Conference, 1998, vol. 2, pp. 907-912.

[12] Chen Wei, Huang Xiaosheng and Zheng Juanjuan "Improved winding loss theoratical calculation of magnetic component with air gap", 7th International Power Electronics and Motion Control Conference, 2012, vol. 1, pp. 471-475.

[13] Fermin A. Holguin, R. Asensi, R. Prieto and J.A. Cobos, "Simple analytical approach for the calculation of winding resistance in gapped magnetic components", 29th Annual IEEE Applied Power Electronics Conference and Exposition, 2014, pp. 2609-2614.

[14] Fermin A. Holguin, R. Prieto, R. Asensi and J.A. Cobos, "Power losses calculations in windings of gapped magnetic components", 29th Annual IEEE Applied Power Electronics Conference and Exposition, 2014, pp. 2605-2608.

[15] Fermin A. Holguin, R. Prieto, R. Asensi and J.A. Cobos, "Power losses calculations in windings of gapped magnetic components: The extended 2D method", in 30th Annual IEEE Applied Power Electronics Conference and Exposition proceedings, 2015.

[16] J. Lammeraner and M. Stalf "Eddy Currents", ILIFFE books LTD., 1966 , chapter 6 and 7.

[17] R. Prieto, J. A. Oliver and J. A. Cobos, "Study of non-axisymmetric magnetic components by means of 2D FEA solvers", IEEE 36 th Power Electronics Specialists Conference, 2005, pp. 1074-107.
[18] M. Albach and H. Roßmanith, "The influence of air gap size and winding position on the proximity losses in high frequency transformers", 32th IEEE Power Electronics Specialists Conference, vol. 3, pp. 1485-1490, 2001.

[19] M. Albach, "Two-dimensional calculation of winding losses in transformers", 31st IEEE Power Electronics Specialists Conference, vol. 3, pp. 1639-1644, 2000.

[20] C.R. Sullivan, "Computationally Efficient Winding Loss Calculation with Multiple Windings, Arbitrary Waveforms and Two- or ThreeDimensional Field Geometry", IEEE Transactions on Power Electronics, vol. 16, no. 1, pp. 142-150, 2001.

[21] D.R. Zimmanck and C.R. Sullivan, "Efficient calculation of windingloss resistance matrices for magnetic components", 12th IEEE Workshop on Control and Modeling for Power Electronics (COMPEL), vol., no., pp. $1,5,28-30,2010$.

[22] J. Acero, P.J. Hernandez, J.M. Burdio, R. Alonso and L.A. Barragdan, "Simple resistance calculation in litz-wire planar windings for induction cooking appliances", IEEE Transactions on Magnetics, vol.41, no.4, pp. $1280-1288,2005$.

[23] J. Acero, R. Alonso, J.M. Burdio, L.A. Barragan and D. Puyal, "Frequency-dependent resistance in Litz-wire planar windings for domestic induction heating appliances", IEEE Transactions on Power Electronics, vol.21, no.4, pp.856-866, 2006.

[24] E.C. Snelling, Soft ferrites, properties and applications, second edition: Butterwoths, 1988.

[25] P. Wallmeier, "Improved analytical modeling of conductive losses in gapped high-frequency inductors", IEEE Transactions on Inchistry Applications, vol. 37, no. 4, pp. 1045-1054, 2001.

[26] Alex Van den Bossche and Vencislav Cekov Valchev, Inductors and Transformers for Power Electronics: Taylor and Francis, 2005.

[27] P. Wallmeier, "Improved analytical modeling of conductive losses in gapped high-frequency inductors", IEEE Transactions on Inahstry Applications, vol. 37, no. 4, pp. 1045-1054, 2001.

[28] Alex Van den Bossche and Vencislav Cekov Valchev, Inductors and Transformers for Power Electronics: Taylor and Francis, 2005.

[29] K. Venkatachalam, C. R. Sullivan, T. Abdallah and H. Tacca, "Accurate prediction of ferrite core loss with nonsinusoidal waveforms using only Steinmetz parameters", IEEE Workshop on Computers in Power Electronics. Proceedings, 2002, pp. 36-41. 